\title{
Total kalça artroplastisinde femoral komponent özellikleri
}

\author{
Properties of the femoral components in total hip arthroplasty
}

\author{
Burak Beksaç, Vahit Emre Özden, İ. Remzi Tözün
}

Acıbadem Üniversitesi Tıp Fakültesi, Ortopedi ve Travmatoloji Bölümü, Acıbadem Maslak Hastanesi, İstanbul

Bu yazıda total kalça artroplastisinde güncel olarak kullanılan femoral komponentlerin tasarım ve kullanım özellikleri anlatılmaktadır. Femoral komponent özellikleri, kullanım şekilleri (çimentolu/çimentosuz), geometrik tasarım özellikleri ve yüzey yapısı, tasarım özelliklerine göre bir sınıflama takip edilerek özetlenmiştir.

Anahtar sözcükler: total kalça replasmanı; femur başı protezi
This paper outlines the design and usage properties of the femoral components currently available in total hip arthroplasty. Cemented and uncemented femoral components' properties will be summarized following the design philosophy, geometry and surface coating types.

Key words: total hip replacement; femoral head prosthesis

oluşumunu ve osteolitik sürecin başlamasını engeller. Bu tip komponentlerin kullanıldığı serilerde Della Valle ve ark. ${ }^{[4]}$ ortalama $6,1(4-8,5)$ sene takipli 73 TKA'nın aseptik gevşeme oranını \%0, Ranawat ve ark. ${ }^{[5]} 9$ sene takipli 236 TKA'nın aseptik gevşeme oranını \%3,4 olarak bildirmişlerdir. Yayımlanan en uzun takipte ise, Callaghan ve ark. $\left.{ }^{1}\right]$ Charnley protezi kullanılan 35 sene takipli seride \%78 sağkalım bildirmişlerdir. Çimentolu femoral komponentin başarısı için, nötral dizilimde yerleştirilmiş bir femoral komponent ve etrafinda en az $2 \mathrm{~mm}$ kalınlığında, bütünlüğü tam bir çimento mantosu gerekli şartlardır.

Mat yüzey özellikli, dikdörtgen kesitli femoral komponentler ise, komponent çimento tutulumunu arttırmak amacıyla çeşitli oranlarda pürüzlü (veya kaplanmış) bir yapıya sahiptir. Clohisy ve Harris, ${ }^{[5]}$ bu tür bir komponentle uygulanan 10 sene takipli 121 hibrid TKA'nın sadece bir tanesinin aseptik gevşeme nedeniyle revizyon gerektirdiğini, üç tanesinin ise radyolojik olarak gevşek ama asemptomatik olduğunu bildirmişlerdir. Bu tür komponentler hakkında iki ayrı görüş vardır: bazı cerrahlar çimento-komponent arası tutulumun bozulmadan kaldığını ve kuvvetli bir femoral bileşik yapı oluşturarak uzun bir sağkalım sağlayabileceğini savunmaktadır. ${ }^{[7]}$ Karşıt görüşte olan cerrahlar ise bu bağlantının, pürüzlü yapısı nedeniyle,

- Illetişim adresi: Dr. Burak Beksaç, Acıbadem Maslak Hastanesi, Ortopedi ve Travmatoloji Bölümü, Büyükdere Cad. No:40 Sarıyer, İstanbul Tel: 0212 - 3044373 e-posta: bbeksac@gmail.com

- Gelis tarihi: 27 Mart 2013 Kabul tarihi: 15 Temmuz 2013 
kısa sürede parçacık üretmeye başlayacağını ${ }^{[8]}$ ve aşırı bir osteoliz cevabı ile gevşeme olacağını söylemektedirler. ${ }^{[9,10]}$

Anatomik yapıdaki çimentolu femoral komponentler en sık İskandinavya'da kullanılmakta ve başarılı takip sonuçları bildirilmektedir. ${ }^{[10]} \mathrm{Bu}$ tip femoral komponentlerin başarısı, etrafındaki çimento mantosuna ve kemiğe dengeli yük aktarımına bağlanmaktadır.

\section{ÇIMENTOSUZ FEMORAL KOMPONENTLER}

Çimentosuz femoral komponentler, 1970'lerde çimentolu femoral komponentlerin alternatifi olarak, daha uzun süre sağkalım amacıyla geliştirilmiştir. Uygulama prensibi, femoral meduller kanalın oyucu ve/veya raspalar ile hazırlanarak ve femoral komponentin bu alana sıkıştırılarak (press-fit) yerleştirilmesi, kemiğin zamanla protezin pürüzlü yüzeyine tutunmasıdır. Eski bazı komponent tasarımlarının kötü sonuçlarına rağmen, kullanımı gittikçe artan çimentosuz femoral komponent tasarımlarının bir çoğu için 10-15 sene takip aralığında düşük aseptik gevşeme sonuçları bildirilmektedir. ${ }^{[11-13]}$

Bu tip komponentlerin, tasarım özelliği olarak, kemiğin içine veya üzerine tutunabileceği pürüzlü yüzeyleri vardır. Yüzeyin sürtünme özelikleri ve elde edilen birincil stabilite, bu tip komponentlerin başarısı için en önemli parametrelerdir. Kemik ile komponent arasında $40 \mu \mathrm{m}$ 'den fazla hareket, kemiğin komponente tutunmasını negatif olarak etkiler ve fibröz tutunma gelişebilir. Yüzeyin pürüzlü yapısı ince/kaba plazma püskürtme (kumlama), boncuk kaplama veya tel örgü (mesh) olabilir. Kemik tutunma için bu yüzeylerin por aralıkları ideal olarak 100-400 $\mu \mathrm{m}$ olmalıdır.

Çimentosuz femoral komponentlerin başarısı incelenirken, yukarıdaki genel tasarım prensiplerine ek olarak, yapıldığı metal alaşım, geometrik şekil, yüzey kaplamasının özellikleri ve modülarite tartışılmalıdır.

Çimentosuz femoral komponentler en çok iki alaşımdan üretilirler: kobalt krom ve titanyum. Kobalt krom alaşımlı komponentlerle karşılaştırıldığında, titanyum alaşımlı komponentler kortikal kemiğin elastik modulusuna eşit sertlikte olduğundan, teorik olarak daha iyi tutunma ve daha az stres kalkanı (stress shielding - tutunum alanının proksimalinde bölgesel osteoporoz) avantajına sahiptirler. Proksimalden tutunumlu titanyum komponentlerin daha az stres kalkanına sebep olduğu DEXA ile kanıtlanmıştır. Kobalt krom ( $\mathrm{CoCr}$ ) komponentlere örnek olarak Trilock, PCA (Porous Coated Anatomic) ve AML (Anatomic Medullary Locking) verilebilir. Bu tip komponentler ile uyluk önü ağrısının daha sık görülmesi, kemik ile komponentin elastik modulus farklılığına bağlanmıştır. Kawamura ve ark. 10 sene takip ettikleri CoCr PCA protezlerinde $\% 36$, Bourne ve ark. ${ }^{[14]} 5$ sene takipte $\% 27$, Healy ve ark. ${ }^{[15]} 12$ sene takipte $\% 25,6$ uyluk önü ağrısı bildirmişlerdir. Tümü kaplı AML protezleri için bildirilen uyluk önü ağrısı oranı \%7-21 arasındadır. ${ }^{[13,16]}$ Ancak yapım alaşımı, kaplama tipi ve stress kalkanı miktarı ne olursa olsun kobalt krom ve titanyum komponentlerin sağkalım süreleri eşdeğer olarak bildirilmektedir.

Çimentosuz femoral komponentler geometrik şekil olarak, kama tipi, silindirik ve anatomik olarak sınıflanabilir ama birçok yeni kuşak komponent bu geometrik tarzların bir karışımı olarak tasarlandığı için kesin bir sınıfa girmeyebilir.

Kama tipi komponentler metafizyel bölgeden kemiğe tutunum sağlayıp, diafizden az veya hiç tutunum prensibine göre tasarlanmıştır. Proksimal kısımda poroz veya kumlanmış yüzey yapısıyla, birincil stabilite ve sonrasında kemik tutunumu sağlanır. Bu komponentler yakasız olarak üretilir ve kemiğe proksimalden bir kama gibi sıkışarak, gelen yükü paylaşır. Bu komponentlerin yük iletimini proksimal femoral bölgeden yapmasının stres kalkanı ve kemik tutulumunun dengelenmesi için en uygun tasarım olduğu söylenmektedir. Bu tür ikinci kuşak tasarımlarda tüm yüzeyi kumlama (titanyum plazma sprey) komponentler de üretilmiş ve günümüzde kullanılmaktadır. ${ }^{[11]}$ Örnek olarak hem CoCr (Tri-Lock - De Puy) hem de titanyum alaşım (Taperloc - Biomet, Warsaw, IN; Synergy, SL Plus - Smith \& Nephew, Memphis, Tenn; CLS Spotorno, Alloclasic Zweymueller - Zimmer, Warsaw, IN) komponentler verilebilir. On sene üzeri takiplerinde çok düşük aseptik gevşeme, uyluk önü ağrısı ve revizyon oranları bildirilen bu tip protezlerin, 20 sene ve üzeri başarılı sonuçları da yayımlanmaya başlamıştır. ${ }^{[11,12,17-19]}$

Silindirik femoral komponentler, poroz yüzey kaplaması çoğunlukla metafizin altına inen ve diafizden tutulum sağlayan tasarımlardır. Birincil tespit, poroz yapının diafize sıkıca oturmasıyla gerçekleşir. Femurun diafizi bu tip protezlerin silindirik yapılarına uygun şekilde oyularak, poroz kaplama uzun bir segmentte kemik endosteal yüzey ile temasa getirilmeye çalışılır. Bu teknik ile komponente rotasyon vermek, gerekirse yüksekte bırakarak uzatma yapmak mümkün olabilir. Bu tip komponentler femurun anatomik eğriliklerine uyum sağlayacak şekilde anatomik olarak tasarlanmıştır. AML (Anatomic Medullary Locking - DePuy, Warsaw, IN) protezi bu tasarımın en bilinen CoCr alaşım örneğidir. Literatürde iyi ve mükemmel uzun takip sonuçları olduğu gibi[16] \%20'lere varan, yüksek uyluk önü ağrısı ve proksimal stres kalkanı oluşumu bildiren yayınlar da vardır. ${ }^{[14]}$ 
Anatomik femoral komponentler, femur metafiz ve diafizine geometrik olarak tam uyum sağlayan, çeşitli uzunluklarda proksimal poroz kaplı (PCA - Stryker Howmedica Osteonics, Allendale, NJ; Anatomic Zimmer, Warsaw, IN) komponentlerdir. Birincil stabilite metafizi, hem koronal hem de sagittal planda tam olarak doldurmalarıyla sağlanır. Teorik olarak doğru bir tasarım olsa da, diğer tip femoral komponentlere karşı bir üstünlükleri gösterilememiştir. Ayrıca, endosteal kemiğe tam temas yüzünden olduğu düşünülen uyluk önü ağrısı komplikasyonu daha fazla olarak bildirilmiştir.

Birincil TKA'da, proksimal femoral deformite ve gelişimsel kalça displazisi gibi özel durumlar dışında çok fazla gerek duyulmayan diğer bir tasarım ise modüler femoral komponentlerdir. Bu tasarımlarda birincil tespit, metafizer kemiğin en sağlam olduğu anatomiye uygun yerleştirilen metafizer parça ile sağlanır. Bu parçanın içine yerleşen bir sap, anteversiyon ve ofseti bunlardan bağımsız olarak ayarlanabilecek bir boyun parçası, modüler olarak sistemi tamamlar. Bu tasarıma örnek olarak (S-ROM - DePuy - Johnson \& Johnson, Warsaw; IN, Emperion - Smith \& Nephew, Memphis, Tenn.) protezleri gösterilebilir. Zor vakalarda teknik olarak kolaylık sağlasa da, maliyetin ve envanterin yüksek olduğu ve monoblok komponentlere göre daha uzun sağkalım bildirilmediği akılda tutulmalıdır. Ayrıca modüler bağlantı bölgelerinde korozyon ve buna bağlı olarak hipersensitivite reaksiyonu ile erken gevşeme gelişebileceği bildirilmiştir. ${ }^{20,21]}$

Son senelerde femoral kanalın anatomik olarak yüklenmesi, stres kalkanının engellenmesi ve özellikle genç hastalarda kemik koruyucu cerrahi prensibi öne çıkmıştır; bu prensibe istinaden femoral boynu koruyan birçok metafizyel kısa komponent tasarımı (Proxima - DePuy, Warsaw, IN; Nanos - Smith \& Nephew, Memphis, Tenn.) piyasaya çıkmıştır. ${ }^{[22-28]}$ Kısa dönem takipli bu çalışmalar umut verici olsa da, bu tip protezlerin cerrahi tekniğe daha bağımlı olduğu ve eğer iyi yerleştirilmez ise yüksek oranda erken mekanik gevşeme görülebileceği bildirilmiştir. Sonuç olarak, çimentosuz femoral komponentlerin kullanımı giderek artmaktadır. Bu artış, kolay kullanım ve değişik klinik senaryolara uyarlanabilirlik sağlayan farklı ve çeşitli tasarımların geliştirilmiş olmasına bağlıdır. Kuşaktan kuşağa geliştirilen bu özelliklerle, mükemmel uzun takip sonuçları sağlanmıştır.

\section{KAYNAKLAR}

1. Callaghan JJ, Bracha P, Liu SS, Piyaworakhun S, Goetz DD, Johnston RC. Survivorship of a Charnley total hip arthroplasty. A concise follow-up, at a minimum of thirtyfive years, of previous reports. J Bone Joint Surg Am 2009;91(11):2617-21. CrossRef
2. Hook S, Moulder E, Yates PJ, Burston BJ, Whitley E, Bannister GC. The Exeter Universal stem: a minimum ten-year review from an independent centre. J Bone Joint Surg Br 2006;88(12):1584-90.

3. Burston BJ, Barnett AJ, Amirfeyz R, Yates PJ, Bannister GC. Clinical and radiological results of the collarless polished tapered stem at 15 years follow-up. J Bone Joint Surg $\mathrm{Br}$ 2012;94(7):889-94. CrossRef

4. González Della Valle A, Comba F, Zoppi A, Salvati EA. Favourable mid-term results of the VerSys $C T$ polished cemented femoral stem for total hip arthroplasty. Int Orthop 2006;30(5):381-6.

5. Rasquinha VJ, Ranawat CS. Durability of the cemented femoral stem in patients 60 to 80 years old. Clin Orthop Relat Res 2004;(419):115-23.

6. Clohisy JC, Harris WH. Primary hybrid total hip replacement, performed with insertion of the acetabular component without cement and a precoat femoral component with cement. An average ten-year follow-up study. J Bone Joint Surg Am 1999;81(2):247-55.

7. Rasquinha VJ, Ranawat CS, Dua V, Ranawat AS, Rodriguez JA. A prospective, randomized, double-blind study of smooth versus rough stems using cement fixation: minimum 5-year follow-up. J Arthroplasty 2004;19(7 Suppl 2):2-9.

8. Meding JB, Nassif JM, Ritter MA. Long-term survival of the T-28 versus the TR-28 cemented total hip arthroplasties. J Arthroplasty 2000;15(7):928-33.

9. González Della Valle A, Rana A, Nestor B, Bostrom M, Westrich G, Salvati EA. Metallic shedding, surface finish changes, and extensive femoral osteolysis in the loose Spectron EF stem. Clin Orthop Relat Res 2006;442:165-70.

10. Espehaug B, Furnes O, Engesaeter LB, Havelin LI. 18 years of results with cemented primary hip prostheses in the Norwegian Arthroplasty Register: concerns about some newer implants. Acta Orthop 2009;80(4):402-12. CrossRef

11. Hwang KT, Kim YH, Kim YS, Choi IY. Total hip arthroplasty using cementless grit-blasted femoral component: a minimum 10-year follow-up study. J Arthroplasty 2012;27(8):1554-61. CrossRef

12. Grübl A, Chiari C, Gruber M, Kaider A, Gottsauner-Wolf F. Cementless total hip arthroplasty with a tapered, rectangular titanium stem and a threaded cup: a minimum ten-year follow-up. J Bone Joint Surg Am 2002;84-A(3):425-31.

13. Loughead JM, O'Connor PA, Charron K, Rorabeck $\mathrm{CH}$, Bourne RB. Twenty-three-year outcome of the porous coated anatomic total hip replacement: a concise follow-up of a previous report. J Bone Joint Surg Am 2012;94(2):151-5. CrossRef

14. Bourne RB, Rorabeck CH, Ghazal ME, Lee MH. Pain in the thigh following total hip replacement with a porous-coated anatomic prosthesis for osteoarthrosis. A five-year follow-up study. J Bone Joint Surg Am 1994;76(10):1464-70.

15. Healy WL, Casey DJ, Iorio R, Appleby D. Evaluation of the porous-coated anatomic hip at 12 years. J Arthroplasty 2002;17(7):856-63.

16. Engh CA Jr, Mohan V, Nagowski JP, Sychterz Terefenko CJ, Engh CA Sr. Influence of stem size on clinical outcome of primary total hip arthroplasty with cementless extensively porous-coated femoral components. J Arthroplasty 2009;24(4):554-9. CrossRef

17. Zwartele R, Peters A, Brouwers J, Olsthoorn P, Brand R, Doets C. Long-term results of cementless primary total hip arthroplasty with a threaded cup and a tapered, rectangular titanium stem in rheumatoid arthritis and osteoarthritis. Int Orthop 2008;32(5):581-7. 
18. Corten $\mathrm{K}$, Bourne RB, Charron $\mathrm{KD}, \mathrm{Au} \mathrm{K}$, Rorabeck $\mathrm{CH}$. Comparison of total hip arthroplasty performed with and without cement: a randomized trial. A concise follow-up, at twenty years, of previous reports. J Bone Joint Surg Am 2011;93(14):1335-8. CrossRef

19. Danesh-Clough T, Bourne RB, Rorabeck $\mathrm{CH}$, McCalden R. The mid-term results of a dual offset uncemented stem for total hip arthroplasty. J Arthroplasty 2007;22(2):195-203.

20. Goldberg JR, Gilbert JL. In vitro corrosion testing of modular hip tapers. J Biomed Mater Res B Appl Biomater 2003;64(2):78-93.

21. Goldberg JR, Gilbert JL, Jacobs JJ, Bauer TW, Paprosky W, Leurgans S. A multicenter retrieval study of the taper interfaces of modular hip prostheses. Clin Orthop Relat Res 2002;(401):149-61.

22. Lazarinis S, Mattsson P, Milbrink J, Mallmin H, Hailer NP. A prospective cohort study on the short collum femorispreserving (CFP) stem using RSA and DXA. Primary stability but no prevention of proximal bone loss in 27 patients followed for 2 years. Acta Orthop 2013 Feb;84(1):32-9. CrossRef

23. Schmidutz F, GrafT, Mazoochian F, Fottner A, Bauer-Melnyk A, Jansson V. Migration analysis of a metaphyseal anchored short-stem hip prosthesis. Acta Orthop 2012;83(4):360-5. CrossRef
24. Molli RG, Lombardi AV Jr, Berend KR, Adams JB, Sneller MA. A short tapered stem reduces intraoperative complications in primary total hip arthroplasty. Clin Orthop Relat Res 2012;470(2):450-61. CrossRef

25. Lim SJ, Kim SM, Lim BH, Moon XW, Park YS. Comparison of manual rasping and robotic milling for short metaphysealfitting stem implantation in total hip arthroplasty: a cadaveric study. Comput Aided Surg 2012;18(1-2):33-40. CrossRef

26. Lerch $M$, von der Haar-Tran $A$, Windhagen $H$, Behrens $B A$, Wefstaedt P, Stukenborg-Colsman CM. Bone remodelling around the Metha short stem in total hip arthroplasty: a prospective dual-energy X-ray absorptiometry study. Int Orthop 2012;36(3):533-8. CrossRef

27. Kim $\mathrm{YH}, \mathrm{Oh} \mathrm{JH}$. A comparison of a conventional versus a short, anatomical metaphyseal-fitting cementless femoral stem in the treatment of patients with a fracture of the femoral neck. J Bone Joint Surg Br 2012;94(6):774-81. CrossRef

28. Yerasimides JG. Use of the Fitmore (R) hip stem bonepreserving system for the minimally invasive anterior-supine approach in hip replacement. Am J Orthop (Belle Mead NJ) 2010;39(10 Suppl):13-6. 